\title{
Quantitative Analysis by Electron Transmission Measurements in a Scanning Electron Microscope
}

\author{
E. Müller*, T. Volkenandt*, D.Z. Hu**, D.M. Schaadt** and D. Gerthsen* \\ * Laboratory for Electron Microscopy and Center for Functional Nanostructures (CFN), Karlsruhe \\ Institute of Technology (KIT), 76128 Karlsruhe, Germany ** Institute of Applied Physics and Center for \\ Functional Nanostructures (CFN), Karlsruhe Institute of Technology (KIT), 76128 Karlsruhe, Germany
}

Scanning transmission electron microscopy (STEM) in a conventional scanning electron microscope (SEM) equipped with a high-angle annular dark-field (HAADF) detector is a promising technique for imaging and quantitative analysis of sample thickness and even composition if a reference region with known composition is available. Composition analysis is facilitated by dominant atomic number (Z-) contrast under HAADF conditions [1] which is more pronounced at low electron energies. Low electron energies are also preferable for the investigation of materials sensitive to knock-on damage. Images are easily obtained without the need of an elaborate transmission electron microscope and do not require as much experience as in high-energy electron imaging. Using thin samples a high lateral resolution in the order of $1 \mathrm{~nm}$ can be achieved without degradation of the signal-to-noise ratio. Despite these advantages of low kV STEM few examples are found in the literature for quantitative analysis at typical SEM energies [2].

The measurements presented in the following were performed in a combined focused-ion-beam (FIB)/SEM FEI Strata400S microscope which is equipped with a semiconductor STEM detector with several ring-like segments. The detector is positioned below and close to the sample holder. HAADF images were taken by collecting electrons transmitted in a hollow cone between 0.2-0.7 rad. The investigated samples are InxGa1-xAs/GaAs heterostructures which contain InxGa1-xAs layers of $25 \mathrm{~nm}$ thickness embedded in GaAs layers with a thickness of $35 \mathrm{~nm}$. The In-concentrations $\mathrm{x}=10 \%, 20 \%, 30$ $\%, 40 \%, 50 \%$ and $77 \%$ are well known from calibrations during molecular beam epitaxy growth. Wedge-shaped samples with a defined thickness profile are prepared by FIB milling to verify the accordance of experiments and Monte Carlo (MC) simulations.

Due to the small mean free path for electron scattering at low electron energies, interactions are subjected to stochastical processes and depend on several parameters like density, thickness, atomic mass and atomic number of the material. MC simulations reproduce adequately the stochastical interaction processes of the electrons in the sample. For quantification of image information measurements are compared with calculations performed for several stacks of parameters, making assumptions regarding the composition of the sample. The NISTMonte package is used, applying elastic Mott scattering cross sections and the continuous energy loss approximation [3]. Convolution with detector characteristics must be taken into account for the comparison of the experimental data with simulations [4].

Fig. 1.a shows a cross-section perspective of a InGaAs/GaAs heterostructure taken under HAADF conditions which contains InxGa1-xAs layers with $\mathrm{x}=10 \%, 20 \%, 30 \%$ and $40 \%$. Intensity line scans perpendicular to the edge of the sample in the region of known composition (here GaAs) show a characteristic shape and maximum which allows the determination of the local thickness and 
wedge angle (Fig. 1.b). A line scan perpendicular to the layers at a position with known thickness (Fig. 1.a) is performed for quantification of the In-concentration. To calibrate the gray levels, the intensity of the InGaAs layers is always normalized with respect to the intensity of the adjacent GaAs layer. Measured and simulated normalized intensities for layers of different In-concentrations are shown in Fig. 2.b. Although the signal variation is quite small the two curves show good agreement and reproduce the composition of the layers.

[1] B.v.Borries et al., Zeitschrift für Physik A Hadrons and Nuclei 116 (1940) 249.

[2] P.G. Merli et al., Ultramicroscopy 94 (2003) 89.

[3] N.W.M. Ritchie, Surf. Interface Anal. 37 (2005) 1006.

[4] T. Volkenandt et al., Microscopy and Microanalysis, submitted

[5] This work has been performed within the projects A2 and Z of the DFG Research Center for Functional Nanostructures and is further supported by a grant from the Ministry of Science, Research and Arts of Baden-Württemberg (Az: 7713.14-300).
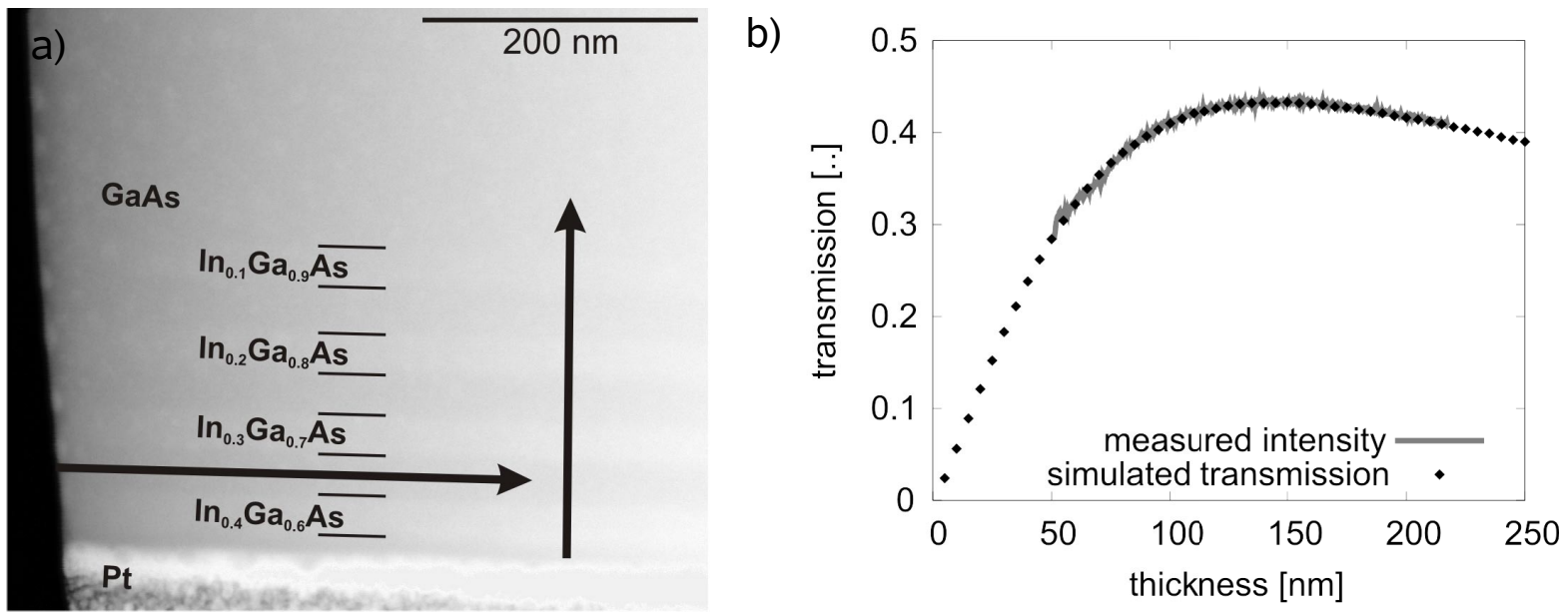

FIG. 1. (a) HAADF STEM image of a $20^{\circ}$ wedge at $25 \mathrm{keV}$. (b) Comparison of the line scan intensity along the GaAs layer with MC simulations as a function of the sample thickness.
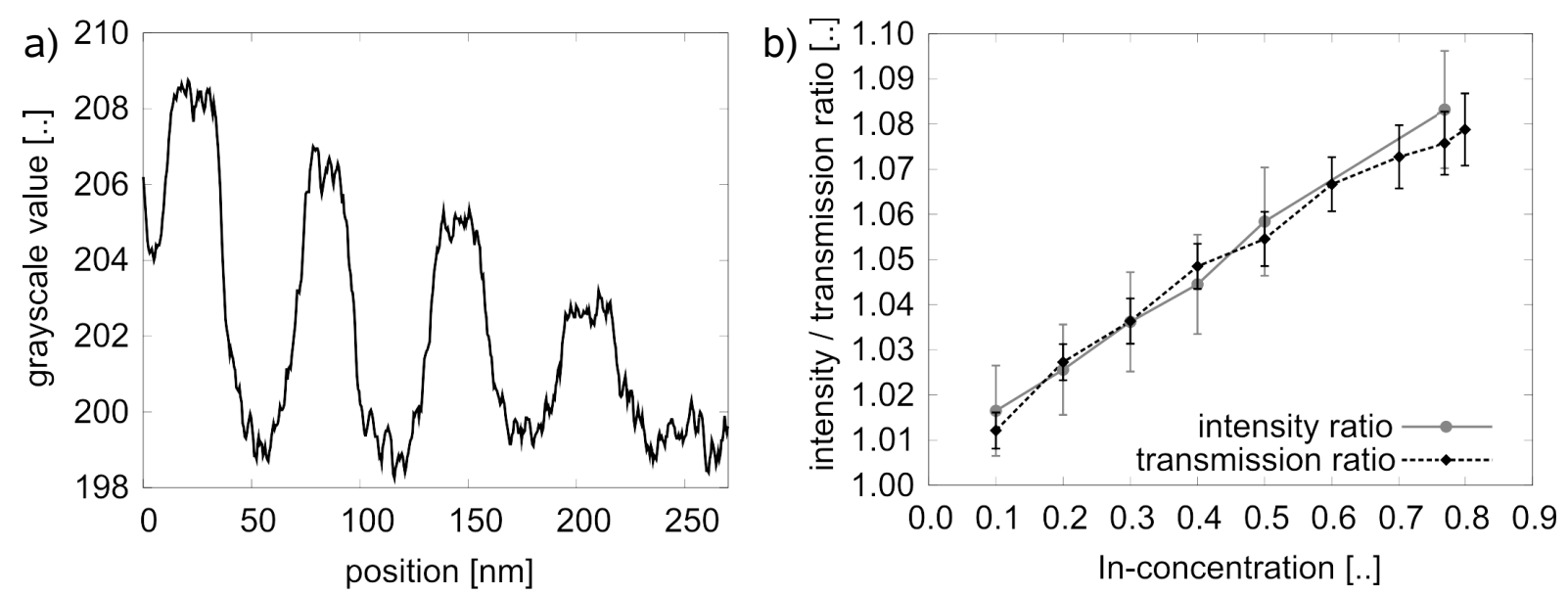

FIG. 2. (a) Grayscale intensities of line scan perpendicular to the $\operatorname{In}_{x} \mathrm{Ga}_{1-\mathrm{x}} \mathrm{As}$ layers (Fig 1a). (b) Comparison of measured normalized intensities with MC simulations as a function of the Inconcentration at a constant sample thickness of $80 \mathrm{~nm}$. 